\title{
SOBRE O MACHO DE ELAVER VALVULA (PICKARD-CAMBRIDGE) (ARANEAE, CLUBIONIDAE)
}

\author{
Antonio D. Brescovit ${ }^{1}$ \\ Alexandre B. Bonaldo ${ }^{2}$
}

\begin{abstract}
ON THE Maie of ElaVer ValvUla (Pickard-Cambridge) (ARANEae, ClubionidaE). The male of Elaver valvula (Pickard-Cambridge) was deseribed by Chickering under the name Clubiona balboae, syn.n.. This species is herein synonymyzed with $E$. valvula and the male is redescribed.

KEY WORDS. Araneae, Clubionidae, Elaver, taxonomy
\end{abstract}

Elaver valvula (Pickard-Cambridge, 1900) foi descrita no gênero Aysha Keyserling, 1891 (Anyphaenidae) com base em fêmea coletada no Panamá. BRESCOVIT \& BONALDO (1992) transferiram esta espécie para o gênero Clubionoides Edwards, 1958, redescrevendo a fêmea e incluindo em sua sinonímia Clubiona silvatica Chickering, 1937, também baseado em uma fêmea do Panamá. BRESCOVIT et al. (1994) transferiram a espécie para Elaver.

Durante visita do autor sênior ao Instituto Nacional de Pesquisas da Amazônia, foram identificados machos e fêmeas de $E$. valvula, provenientes da Ilha de Maracá (Roraima), constatando-se que estes são co-específicos com os machos do Panamá descritos como Clubiona balboae Chickering, 1937.

Coleções examinadas (curadores entre parênteses): BMNH - The Natural History Museum, Londres, Inglaterra (P.D. Hillyard); INPA - Instituto Nacional de Pesquisas da Amazônia, Manaus, Brasil (C. Magalhães); MCN - Museu de Ciências Naturais, Fundação Zoobotânica do Rio Grande do Sul, Porto Alegre, Brasil (E.H. Buckup); MCZ - Museum of Comparative Zoology, Cambridge, Mass., EUA (H.W. Levi).

O formato da descrição e as abreviaturas são os mesmos usados por BRESCOVIT \& BONALDO (1992). Todas as medidas são expressas em milímetros $(\mathrm{mm})$.

1) Laboratório de Artrópodos Peçonhentos, Instituto Butantan, Av. Vital Brasil 1500, Caixa Postal 65 , 05503-900 São Paulo, São Paulo, Brasil.

2) Museu de Ciências Naturais, Fundação Zoobotânica do Rio Grande do Sul, Caixa Postal 1188 , 90001-970 Porto Alegre, Rio Grande do Sul, Brasil. Bolsista da CAPES. 


\section{Elaver valvula (Pickard-Cambridge, 1900)}

Figs 1-3

Aysha valvula Pickard-Cambridge, 1900: 99, pr. 7, fig. 20 (holótipo 9 BMNH 321, Bugaba, Chiriqui, Panamá) [Examinado].

Clubiona balboae Chickering, 1937: fig. 4, 77-79 (holótipo ơ MCZ. Barro Colorado Island, Panamá) [Examinado]. Syn.n..

Clubiona silvatica Chickering, 1937: 20, figs 12, 25 (holótipo 9 MCZ, Barro Colorado Island, Canal Zone, Panamá) [Examinado].- Brescovit \& Bonaldo, 1992: 689.

Clubiona balboai: Bonnet, 1956: 1110.

Clubionoides valvula: Brescovit \& Bonaldo, 1992: 689, figs. 8-9.

Clubionoides balboai: Brescovit \& Bonaldo, 1992: 689.

Elaver balboae: Brescovit, Bonaldo \& Mikhailov, 1994: 36.

Elaver silvatica: Brescovit, Bonaldo \& Mikhailov, 1994: 37.

Elaver valvula: Brescovit, Bonaldo \& Mikhailov, 1994: 38.

Diagnose. O macho aqui descrito é semelhante ao de E. brevipes (Keyserling, 1891) - ver BRESCOVIT \& BONALDO (1992), figs. 1-2 para comparação - por apresentar projeção tegular basal. Difere pela orientação retrolateral desta estrutura e pela apófise tibial retrolateral simples, não bífida (Figs 1-3).

Descrição. Macho. Ilha de Maracá (Roraima). Coloração como na fêmea. Comprimento total 4,40. Carapaça: comprimento 2,10, largura 1,50. Clípeo: altura 0,06. Olhos: diâmetros, OMA 0,13 , OLA 0,14, OMP 0,13, OLP 0,13 . Interdistâncias: OMA-OMA 0,06, OMA-OLA 0,04, OMP-OMP 0,15, OMP-OLP 0,11, OLA-OLP 0,05. QOM: comprimento 0,32, largura anterior 0,31 , largura posterior 0,51 . Quelíceras: cinco dentes na promargem e cinco dentículos na retromargem.

Pernas - I: fêmur 1,50 , patela 0,70 , tíbia 0,40 , metatarso 1,00 , tarso 0,50 , total 5, 10; II: $1,70,0,80,1,70,1,20,0,60,6,00$; III: $1,30,0,55,1,00,2,00,0,40$, 4,45 ; IV: $1,75,0,75,1,60,2,00,0,50,6,60$. Espinulação: pernas I - fêmur d1-1-1, rd0-1-1, pd2, tíbia v2-2-0, p0, r0, metatarso v2-0-0, p0, r0; II: fêmur d1-1-1, rd0-1-1, pd1-1, tíbia v2-2-0, p0, r0, metatarso v2-0-0, p0, r0; III - IV - fêmur d1-1-1, pd0-1-1, rd0-1-1, tíbia v1p-1p-2, p1-1-0, r1-1-0, metatarso v2-2-2, p1-1-1, r1-1-1. Palpo: figs 1-3.

Distribuição geográfica. Panamá e norte do Brasil (Roraima).

Material examinado. PANAMÁ, Canal Zone: Barro Colorado Island, uma fêmea (MCZ, holótipo). BRASIL, Roraima: Alto Alegre, (Rio Uraricoera, Ilha de Maracá, Estação Ecológica de Maracá), um macho, 22. VIII.1987, R. Gribel leg. (MCN 19755); um macho, 19.III.1987, uma fêmea, 4.XII.1987, uma fêmea, 10.XII.1987, A.A. Lise leg. (INPA).

AGRADECIMENTOS. Ao Dr. Herbert W. Levi pelo empréstimo do tipo de E. balboai; ao Dr. C. Magalhães (INPA) pelo acesso à coleção de aranhas e à pesquisadora Erica H. Buckup (MCN) pelas sugestōes. 

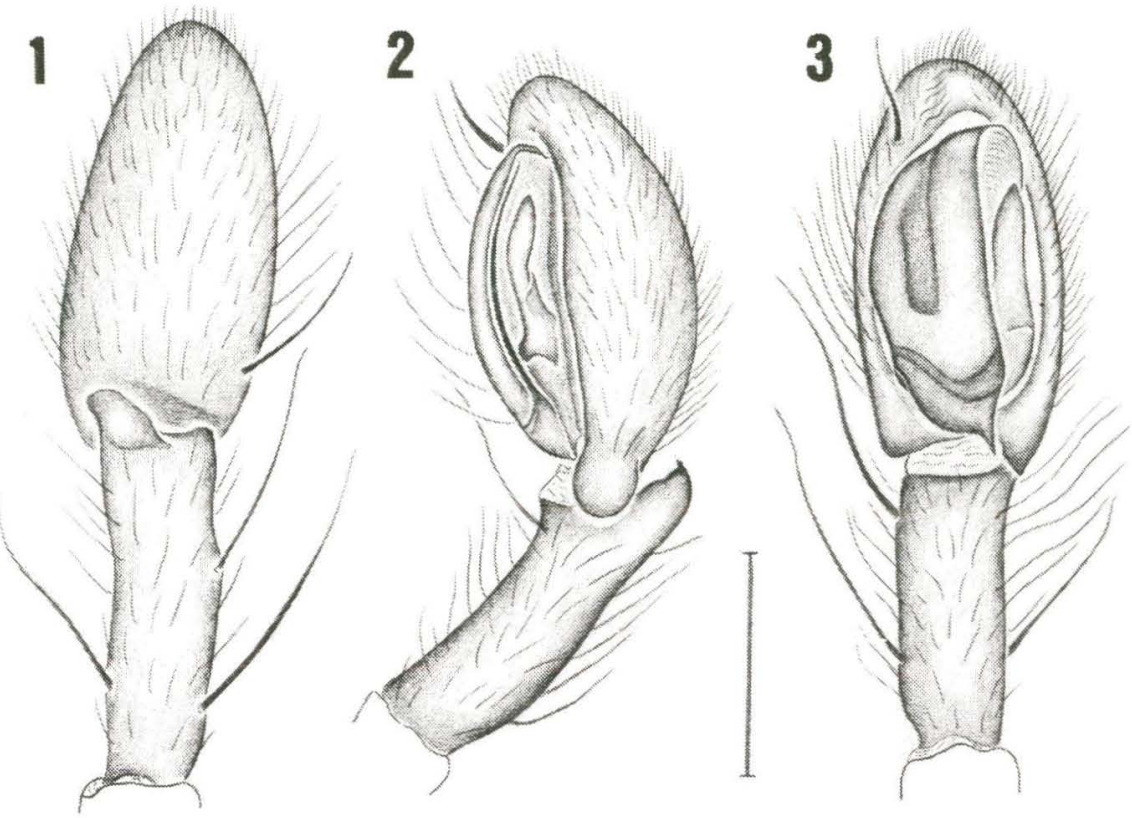

Figs 1-3. Elaver valvula (Pickard-Cambridge), palpo do macho (MCN 19755). (1) Vista dorsal; (2) vista retrolateral: (3) vista ventral. Escala: $0.25 \mathrm{~mm}$.

\section{REFERÊNCIAS BIBLIOGRÁFICAS}

Bonnet, P. 1956. Bibliographia Araneorum. Toulouse, Douladoure, 2 (2): 919-1926.

BResCovit, A.D. \& A.B. Bonaldo. 1992. Gênero Clubionoides Edwards, 1958 (Araneae, Anyphaenidae): combinações novas e redescrição de quatro espécies neotropicais. Revta bras. Ent. 36 (3): 685-692.

Brescovit, A.D.; A.B. Bonaldo \& K. Mikhailov. 1994. Revalidation of the genus Elaver O. Pickard-Cambridge, 1898 (Araneae, Clubionidae). Arthropoda Selecta, Moscou, 3 (1-2): 35-38.

Chickering, A.M. 1937. The Clubionids of Barro Colorado Island, Panamá. Trans. Am. microsc. Soc. 56: 1-47.

PiCKARD-CAMBridge, F.O. 1900. Arachnida-Araneida, p. 89-194. In: F.D. Godman \& O. SAlvin (ed.). Biologia Centrali Americana. London, 2: 409-610. 